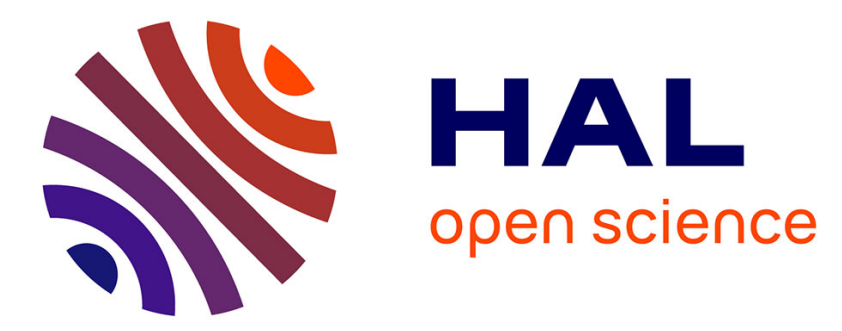

\title{
Synthesis of CdSe Nanoplatelets without Short-Chain Ligands: Implication for Their Growth Mechanisms
} Ye Jiang, Wilfried-Solo Ojo, Benoit Mahler, Xiangzhen Xu, Benjamin Abécassis, Benoît Dubertret

\section{- To cite this version:}

Ye Jiang, Wilfried-Solo Ojo, Benoit Mahler, Xiangzhen Xu, Benjamin Abécassis, et al.. Synthesis of CdSe Nanoplatelets without Short-Chain Ligands: Implication for Their Growth Mechanisms. ACS Omega, 2018, 3 (6), pp.6199 - 6205. 10.1021/acsomega.8b01006 . hal-01889569

\section{HAL Id: hal-01889569 https://hal.science/hal-01889569}

Submitted on 9 Nov 2020

HAL is a multi-disciplinary open access archive for the deposit and dissemination of scientific research documents, whether they are published or not. The documents may come from teaching and research institutions in France or abroad, or from public or private research centers.
L'archive ouverte pluridisciplinaire HAL, est destinée au dépôt et à la diffusion de documents scientifiques de niveau recherche, publiés ou non, émanant des établissements d'enseignement et de recherche français ou étrangers, des laboratoires publics ou privés. 


\title{
Synthesis of CdSe Nanoplatelets without Short-Chain Ligands: Implication for Their Growth Mechanisms
}

\author{
Ye Jiang, ${ }^{\dagger}$ Wilfried-Solo Ojo, ${ }^{\ddagger}$ Benoit Mahler, ${ }^{\S}$ Xiangzhen Xu, ${ }^{\dagger}$ Benjamin Abécassis, ${ }^{*}, \|, \perp_{\odot}$ \\ and Benoit Dubertret* ${ }^{\dagger} \dagger$ \\ ${ }^{\dagger}$ Laboratoire de Physique et d’Etude des Materiaux, CNRS, UMR 8213, ESPCI ParisTech, 10 Rue Vauquelin, 75005 Paris, France \\ ${ }^{\ddagger}$ Nexdot, 102 Avenue Gaston Roussel, 93230 Romainville, France \\ ${ }^{\S}$ Institut Lumière Matière, CNRS, UMR 5306, 10 Rue Ada Byron, 69100 Villeurbanne, France \\ "Laboratoire de Chimie, ENS de Lyon, CNRS, UMR 5182, 46 Allée d’Italie, 69364 Lyon, France \\ ${ }^{\perp}$ Laboratoire de Physique des Solides, Université Paris-Saclay, CNRS, UMR 8502, Université Paris-Sud, 91405 Orsay, France
}

Supporting Information

ABSTRACT: We present a novel route for the synthesis of zinc blende CdSe nanoplatelets (NPLs) that exclude the use of shortchain alkyl carboxylates. CdSe NPLs obtained without acetates are shown to be extremely asymmetric and rectangular. The effects of several experimental parameters such as the nature of cadmium carboxylates, selenium precursors, and precursor concentration ratios are studied. Our experiments, together with complementary small-/ wide-angle X-ray scattering results, show that the formation of NPLs is not related to soft templating. We discuss our findings in regard to several other formation mechanisms of NPLs, which have appeared recently in the literature, and propose that the steric hindrance caused by ligand packing exerts an influence on the growth and geometry of two-dimensional NPLs.

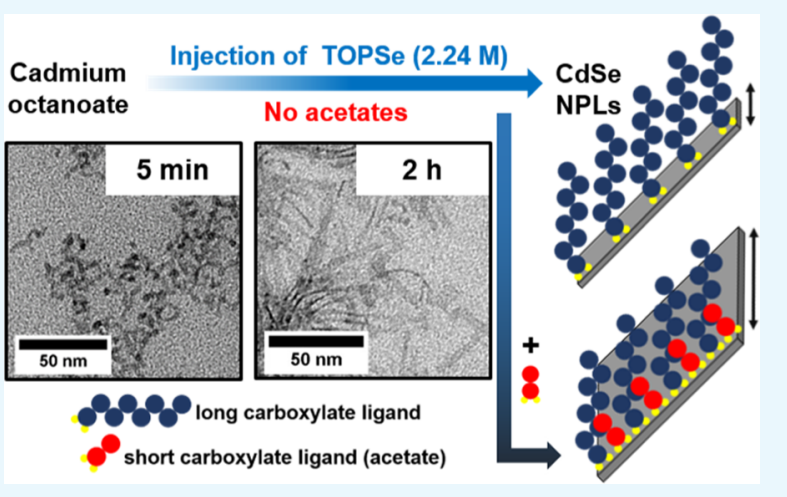

\section{INTRODUCTION}

The colloidal synthesis of freestanding two-dimensional (2D) nanostructures has been an attractive topic because of their unique physical and chemical properties. ${ }^{1,2}$ For instance, colloidal CdSe nanoplatelets (NPLs) can be synthesized with a thickness controlled with atomic precision, ${ }^{3}$ which results in exceptional optical properties, comparable to the ones offered by the quantum wells synthesized with epitaxial growth techniques. ${ }^{4}$ In these $2 \mathrm{D}$ structures, the density of electron and hole states is a continuous steplike function of the energy, whereas for zero-dimensional or one-dimensional (1D) nanocrystals, it has a discrete nature. ${ }^{5}$ The binding energy of excitons is enhanced greatly because of the small dielectric constant of the surrounding media ${ }^{6}$ and the effect of mirror charges at the interface. ${ }^{7}$ This leads to an extremely narrow photoluminescence (PL) line width and a very short fluorescence lifetime. ${ }^{8}$

The 2D colloidal CdSe nanocrystals have been synthesized in two different crystal structures: hexagonal wurtzite (WZ) CdSe nanoribbons ${ }^{9}$ or quantum belts ${ }^{10}$ and cubic zinc blende (ZB) CdSe NPLs. ${ }^{3}$ Both the growth mechanisms and the final objects are very different for these two crystal structures. In the case of WZ nanoparticles, a lamellar-like template growth with a cadmium chloride alkylamine complex serving as a mold during the formation of WZ CdSe 2D nanostructures has been evidenced. $^{9-13}$ These nanoparticles aggregate easily, have weakly bound ligands, and have a balanced number of anions and cations. On the contrary, CdSe NPLs ${ }^{3}$ with a cubic ZB crystal structure have an excess of cations, controllable lateral extension from few nanometers to a micrometer ${ }^{14}$ and good colloidal stability, thanks to carboxylate ligands bound onto the two cadmium-rich large base facets. Their thickness can be controlled precisely and different chalcogenide NPLs have been synthesized. ${ }^{4,15}$ The first attempt to describe the formation mechanism of ZB NPLs in 2011 showed that CdSe NPLs are formed through continuous lateral extension of small seeds that possess some facets blocked by the ligands. The thickness of the NPLs is fixed in the very first stages of the formation. ${ }^{16}$ The lateral extension of NPLs through continuous reaction of precursors has been confirmed later when NPLs with lateral dimensions of few tens of nanometers are extended laterally to dimensions of few hundreds of nanometers with continuous injection of precursors. ${ }^{14}$ This lateral extension proceeds without the formation of seeds, which suggests that the NPLs extend through continuous reaction of precursors rather than through oriented attachment, as is the case for rock salt $\mathrm{PbS}$ NPLs. ${ }^{17}$ Recently, three alternative growth mechanisms have been proposed to describe the formation of ZB CdSe NPLs. (i)

Received: May 15, 2018

Accepted: May 25, 2018

Published: June 8, 2018 


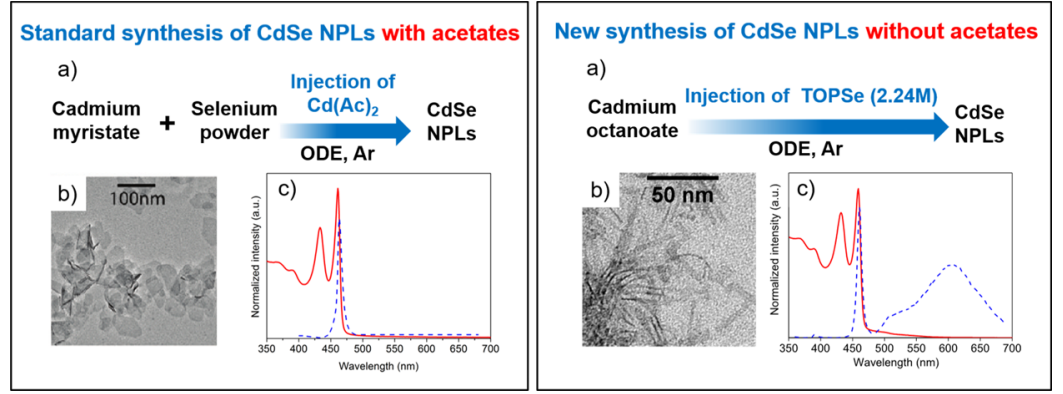

Figure 1. Comparisons between a standard synthesis for ZB CdSe NPLs (left) and the new synthesis without acetate salts (right) with their schematic routes illustrated in (a) correspondingly as well as TEM images and absorption (solid line)/PL (dashed line) spectra in (b,c). This figure is reproduced from refs 3 and 16 .

Table 1. Experimental Parameters Explored for the Synthesis of CdSe NPLs without Acetate Salts

\begin{tabular}{|c|c|c|c|c|c|}
\hline precursors & type of precursor & reaction temperature $/{ }^{\circ} \mathrm{C}$ & \multicolumn{2}{|c|}{ concentration of $\mathrm{Cd}$ precursor $/ \mathrm{mol} / \mathrm{L}$} & molar ratio of precursors $(\mathrm{Se} / \mathrm{Cd})$ \\
\hline \multirow[t]{4}{*}{ Se precursor } & Se powder & 240 & \multirow[t]{8}{*}{$\mathrm{CdC}_{8}$} & 0.1 & $0.2: 1$ \\
\hline & $\mathrm{Se}-\mathrm{ODE}$ & $210,230,250$ & & 0.1 & $0.2: 1$ \\
\hline & TOPSe $(1 \mathrm{M})$ & 150,175 & & 0.1 & $0.2: 1$ \\
\hline & TOPSe $(2.24 \mathrm{M})$ & $150,175,200,225$ & & 0.1 & $0.2: 1$ \\
\hline \multirow[t]{4}{*}{$\mathrm{Cd}$ precursor } & $\mathrm{CdC}_{8}$ & 175 & & $0.1,0.06,0.01,0.005,0.002$ & TOPSe (2.24 M) fixed \\
\hline & $\mathrm{CdC}_{8}$ & 175 & & 0.1 & $0.2: 1,0.3: 1,1: 1$ \\
\hline & $\mathrm{CdC}_{10}$ & 175 & & 0.1 & $0.2: 1$ \\
\hline & $\mathrm{CdC}_{14}$ & 180 & & 0.1 & $0.2: 1$ \\
\hline
\end{tabular}

Oriented attachment: Peng's group has been able to isolate the seeds of $550 \mathrm{~nm}$ emitting NPLs and showed that these seeds form NPLs through oriented attachment. ${ }^{18}$ They suggest that both cadmium acetate and cadmium $n$-alkanoate with large $n$ ( $n$ $>7$ ) are necessary for the conversion of spherical seeds into $2 \mathrm{D}$ NPLs. (ii) Soft templated formation: Lyashchova et al. have shown that CdSe ZB NPLs can be obtained from cadmium octanoate without the addition of short-chain Cd(carboxylate) in a saturated aqueous alcoholic solution of selenourea. ${ }^{19}$ The authors conclude that the NPLs form in the smectic A mesophase of the cadmium octanoate. In fact, mesomorphic phase transitions can be observed for many metal carboxylates, showing thermotropic lamellar or columnar mesophases in the phase sequences. ${ }^{20,21}$ In the case of cadmium carboxylates, the phase diagram depends on the carboxylate chain length. ${ }^{22}$ It has also been found that cadmium myristate in octadecene solution shows a lamellar mesophase before melting into micelles for colloidal synthesis of quantum dots. ${ }^{23}$ Unfortunately, in the work of Lyashchova, no transmission electron microscopy (TEM) images of the NPLs have been shown, and the presence of the lamellar phases has not been linked to the formation of the 2D CdSe NPLs. (iii) Growth driven by precursors' insolubility: Riedinger et al. ${ }^{24}$ have shown that NPLs can form in isotropic melts of either $\mathrm{Cd}$ (myristate) $)_{2}$ or Cd(propionate) $)_{2}$, confirming that a mixture of short and long cadmium carboxylate is not necessary to obtain NPLs. The authors state that the solubility of $\mathrm{Cd}$ (carboxylate) $)_{2}$ in octadecene determines whether quantum dots or NPLs are formed. If the $\mathrm{Cd}$ precursor dissolves, quantum dots are formed; if the Cd precursor is insoluble, NPLs would form.

Here, we study the possibility to synthesize CdSe NPLs in octadecene with pure long-chain cadmium carboxylate precursors without detectable aggregation. We study the effect on the reaction of several different cadmium carboxylates, different selenium precursors, and a wide range of synthetic conditions. UV absorption combined with TEM images of the samples demonstrates that when short alkyl chain carboxylate precursors are not used, the NPLs have extremely asymmetric, rectangular shapes with a small dimension that is often smaller than $10 \mathrm{~nm}$. We observe that for the synthesis of NPLs without cadmium acetate, we need (i) a high concentration of cadmium precursors $(0.1 \mathrm{~mol} / \mathrm{L})$, (ii) selenium precursors with a good reactivity, and (iii) the temperature to be sufficiently high to promote precursors' reactivity. In contrast to Riedinger et al., we show that NPLs can be formed in a soluble mixture of long alkyl chain cadmium precursors with 1-octadecene (ODE). In contrast to Lyashchova et al., we show that NPLs can be formed even in the absence of soft templating. At the onset of the NPL formation, wormlike NPLs are observed with small-/ wide-angle X-ray scattering (SAXS/WAXS) studies of the precursor demonstrating that the lamellar and/or columnar phase of cadmium octanoate persists in solution up to $180{ }^{\circ} \mathrm{C}$ but disappears above $200{ }^{\circ} \mathrm{C}$ under our experimental conditions. In spite of the absence of any molecular templates above $200{ }^{\circ} \mathrm{C}$, NPLs can be formed. At intermediate steps, ex situ electron microscopy does not show evidence of spherical seeds, which rules out an oriented attachment-driven mechanism such as that observed by Peng et al. for thicker NPLs.

\section{RESULTS AND DISCUSSION}

Our synthesis is based on a liquid-phase hot-injection methodology (Figure 1, right). A typical protocol is as follows: in a three-neck flask, $1 \mathrm{mmol}$ of cadmium octanoate $\left(\mathrm{CdC}_{8}\right)$ in $10 \mathrm{~mL}$ of ODE is heated up to $175{ }^{\circ} \mathrm{C}$ under argon flow, and then a mixture of $100 \mu \mathrm{L}$ of stoichiometric TOPSe $(2.24 \mathrm{M})$ in $0.5 \mathrm{~mL}$ of ODE is injected. Two minutes after the injection, the color of the clear solution becomes yellowish. The synthesis reaction can last up to $2 \mathrm{~h}$. The final color of the solution is bright orange with CdSe NPLs suspended in the mixture. The resulting CdSe NPLs are precipitated and washed three times with ethanol and then dispersed in hexane for further 


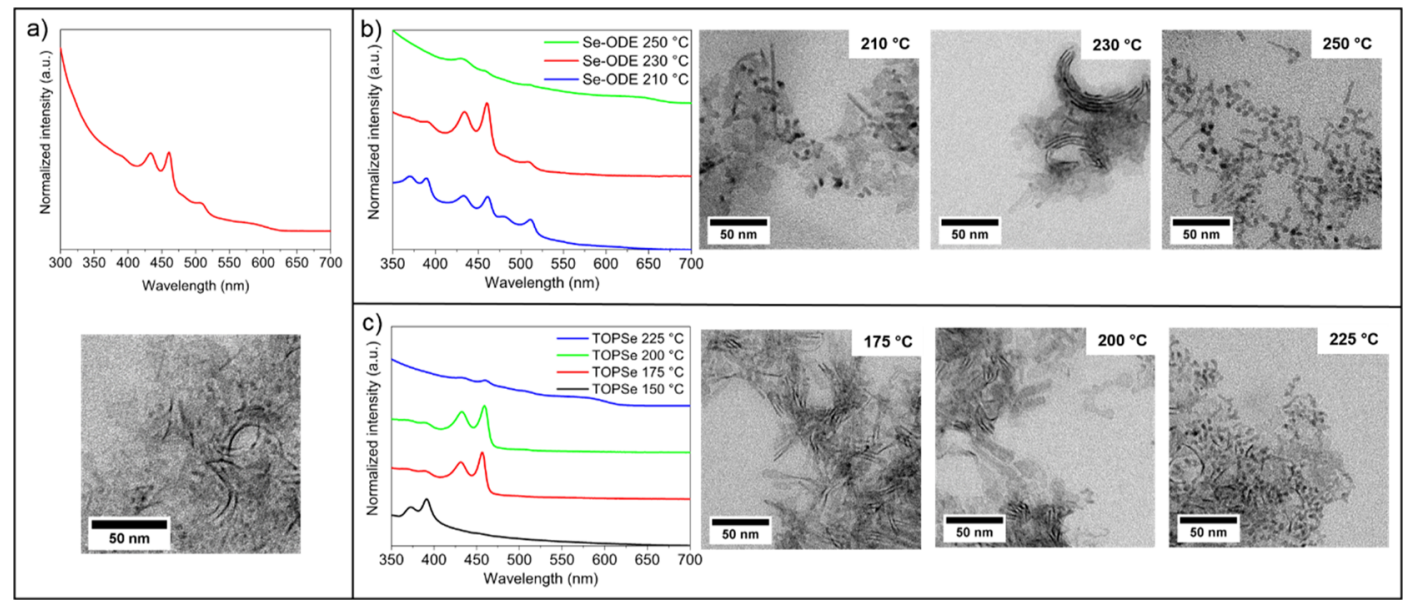

Figure 2. Influence of the selenium precursor on the formation of CdSe NPLs: (a) $0.4 \mathrm{~g}^{\circ} \mathrm{CdC}_{8}$ and $12 \mathrm{mg}$ of selenium powder in $10 \mathrm{~mL}$ of ODE and one-pot heating up to $240{ }^{\circ} \mathrm{C}$ under $\mathrm{Ar}$; (b) $0.4 \mathrm{~g}$ of $\mathrm{CdC}_{8}$ in $10 \mathrm{~mL}$ of ODE, $1 \mathrm{~mL}$ of Se-ODE $(0.1 \mathrm{M})$ injected at different temperatures under $\mathrm{Ar}$; and (c) $0.4 \mathrm{~g}$ of $\mathrm{CdC}_{8}$ in $10 \mathrm{~mL}$ of ODE, $100 \mu \mathrm{L}$ of TOPSe $(2.24 \mathrm{M})$ in $0.5 \mathrm{~mL}$ of ODE injected at different temperatures under Ar.

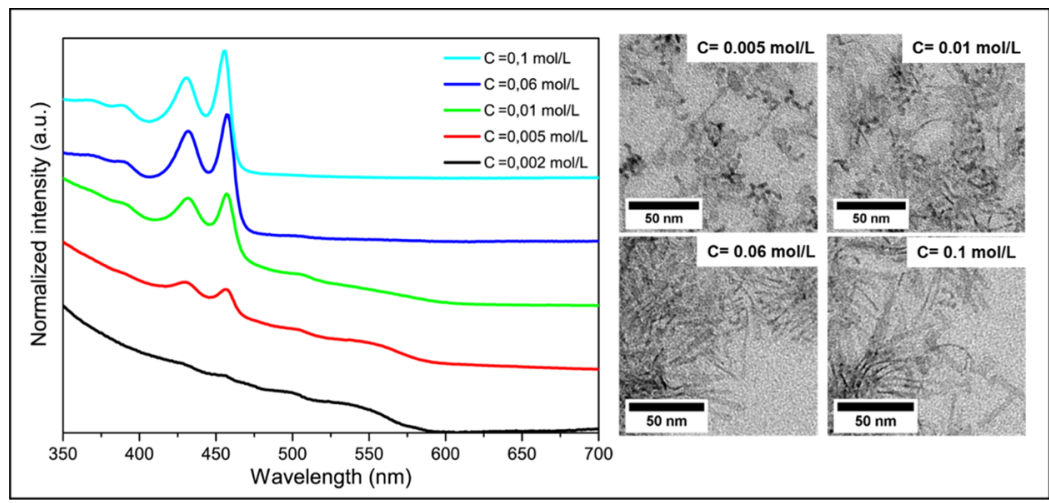

Figure 3. Influence of the concentration of cadmium precursor in ODE: various amounts of $\mathrm{CdC}_{8}$ in $10 \mathrm{~mL}$ of $\mathrm{ODE}$ and $100 \mu \mathrm{L}$ of TOPSe (2.24 M) in $0.5 \mathrm{~mL}$ of $\mathrm{ODE}$ injected at $175^{\circ} \mathrm{C}$ under $\mathrm{Ar}$.

characterizations. The synthesis yields NPLs with typical absorption features of three-monolayer (ML) thick ZB NPLs including two excitonic transition peaks with maxima at 432 and $459 \mathrm{~nm}$, respectively, corresponding to the electron/light hole and the electron/heavy hole transitions. ${ }^{3}$ The PL emission spectrum presents a strong emission peak at $461 \mathrm{~nm}$ with a full width at half-maximum of $15 \mathrm{~nm}$ combined with a broad emission signal at low energy that can be attributed to trap emission. A $2 \mathrm{~nm}$ stoke shift is detected in the case of PL, and the quantum yield is measured to be around 3\%. The TEM of the NPLs shows 2D structures with very asymmetric rectangular-like shapes (Figure S1). These elongated shapes are very different from the much more symmetrical shape of the three-ML NPLs obtained with other protocols., ${ }^{3,26}$ X-ray powder diffraction (XRD) confirmed the ZB crystal structure of the NPLs (Figure S2).

We have explored the different experimental parameters that influence this synthesis (see Table 1). We first study the influence of the precursors of selenium (Figure 2). When Se powder is used, three-ML NPLs can be obtained without acetate salts (Figure 2a). The synthesis follows a one-pot methodology with a high reaction temperature of $240{ }^{\circ} \mathrm{C}$ that is necessary to activate the Se precursor in ODE. We can obtain CdSe NPLs but with very low chemical yields because of the limited reactivity of the Se precursor. Consequently, for the following trials, we focus on two other Se precursors: Se-ODE
$(0.1 \mathrm{M})$ and stoichiometric TOPSe $(2.24 \mathrm{M})$. In these cases, the syntheses were conducted using a hot-injection approach at various reaction temperatures, as detailed in Figure $2 \mathrm{~b}, \mathrm{c}$. Both Se-ODE $(0.1 \mathrm{M})$ and TOPSe $(2.24 \mathrm{M})$ can lead to the formation of CdSe NPLs without the addition of acetate salts. The reason why we treat $\mathrm{CdC}_{8}$ and $\mathrm{Se}-\mathrm{ODE}$ at higher temperatures is that generally the reactivity of $\mathrm{Se}-\mathrm{ODE}$ is not as high as TOPSe, and thus, higher reaction temperatures are required for the reaction to proceed. When $\mathrm{Se}-\mathrm{ODE}$ is used, we observe that NPL formation always comes with the formation of $\mathrm{CdSe}$ quantum dots as indicated by the absorption tail at low energy range in the absorption spectrum. This could be due to the reactions between the cadmium precursor and the various reagents produced from the complex activation process of Se-ODE during the synthesis. ${ }^{27}$ When the reaction temperature is raised from 210 to $230{ }^{\circ} \mathrm{C}$, mostly three-ML thick CdSe NPLs are formed. However, when the temperature is further increased to $250{ }^{\circ} \mathrm{C}$, a considerable decrease in the NPL formation yield is observed rather than the formation of four-ML thick NPLs. When TOPSe $(2.24 \mathrm{M})$ is used as a Se precursor, the same trends occur. A low reaction temperature of $150{ }^{\circ} \mathrm{C}$ produces CdSe NPLs with a thickness of only two MLs. Comparatively, pure three-ML NPLs are obtained when the reaction temperature is raised to $175{ }^{\circ} \mathrm{C}$. For reaction temperatures of $200{ }^{\circ} \mathrm{C}$ or higher, the shape of the CdSe NPLs becomes distorted, and when the temperature is further 


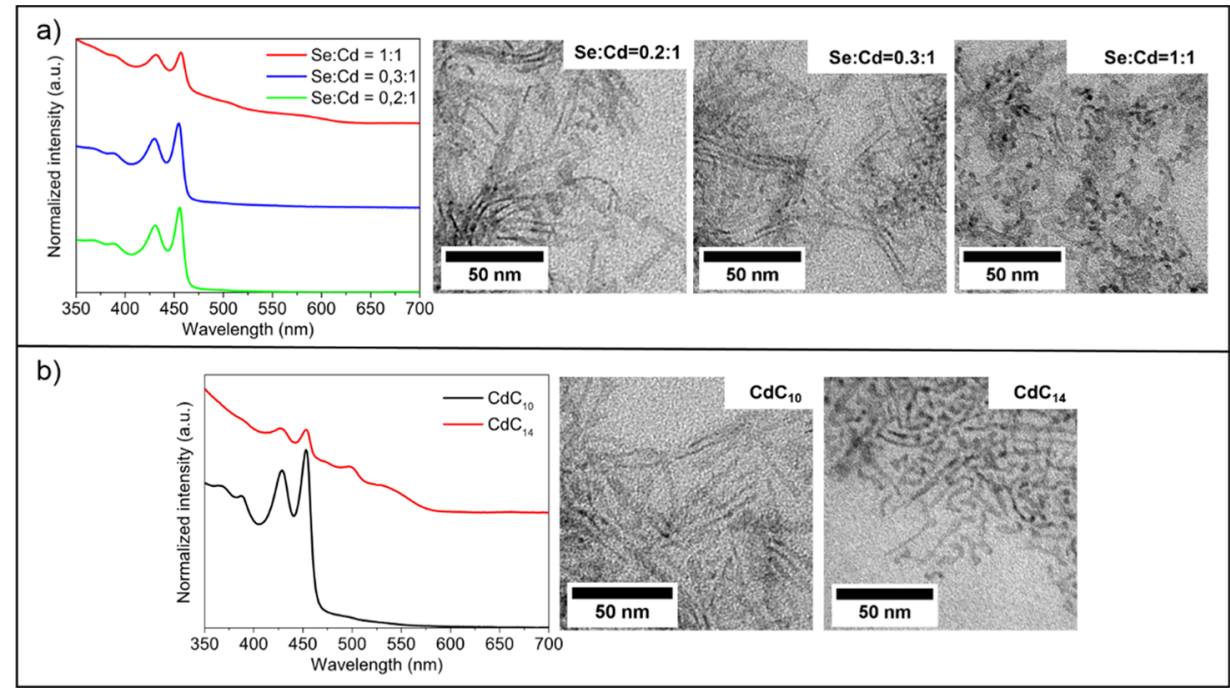

Figure 4. (a) Influence of the ratio of precursors $\mathrm{Se} / \mathrm{Cd}$ for a fixed concentration of the cadmium precursor: $0.4 \mathrm{~g}$ of CdC 8 in $10 \mathrm{~mL}$ of $\mathrm{ODE}$ and various amounts of TOPSe $(2.24 \mathrm{M})$ in $0.5 \mathrm{~mL}$ of ODE injected at $175{ }^{\circ} \mathrm{C}$ under Ar and (b) influence of cadmium carboxylate: $1 \mathrm{mmol}$ of Cd carboxylate in $10 \mathrm{~mL}$ of ODE and $100 \mu \mathrm{L}$ of TOPSe $(2.24 \mathrm{M})$ in $0.5 \mathrm{~mL}$ of ODE injected at $175^{\circ} \mathrm{C}$ under Ar.

increased to $225{ }^{\circ} \mathrm{C}$, the amount of NPLs is significantly reduced. Overall, the NPLs synthesized with Se-ODE have very different shapes with strong shape dispersions, whereas the NPLs synthesized with TOPSe are more rectangular-shaped. It should be mentioned here that $2.24 \mathrm{M}$ TOPSe rather than $1 \mathrm{M}$ TOPSe is used in our syntheses. When $1 \mathrm{M}$ TOPSe is used in place of $2.24 \mathrm{M}$ TOPSe, 1D nanowires are obtained (Figure S3).

We have also studied the influence of the long $n$-alkanoate cadmium precursor on the possibility to obtain NPLs without cadmium acetate. We first focus on cadmium octanoate. We observed that the formation of CdSe NPLs strongly depends on the concentration of the cadmium precursor $\left(\mathrm{CdC}_{8}\right)$ in octadecene $\left(C_{\mathrm{CdC}_{8}}\right)$. We did not observe the absorption features corresponding to NPLs when $C_{\mathrm{CdC}_{8}}$ is as low as $0.002 \mathrm{~mol} / \mathrm{L}$ (Figure 3). The characteristic excitonic peaks of CdSe NPLs begin to appear when $C_{\mathrm{CdC}_{8}}$ reaches $0.005 \mathrm{~mol} / \mathrm{L}$. CdSe NPLs prepared at higher concentrations (e.g., $C_{\mathrm{CdC}_{8}}=0.06 \mathrm{~mol} / \mathrm{L}$ or $0.1 \mathrm{~mol} / \mathrm{L}$ ) have more regular shape and are produced in larger quantity.

We have also investigated the influence of the ratio of precursors $\mathrm{Se} / \mathrm{Cd}$ for a fixed concentration of the cadmium precursor $C_{\mathrm{CdC}_{8}}=0.1 \mathrm{~mol} / \mathrm{L}$. We find that when cadmium is in excess compared to selenium, NPLs are formed more easily in higher yields (Figure 4a). For equimolar concentration of cadmium and selenium, three-ML NPLs can be formed, but a large number of other structures are formed as well (Figure 4a, right). The necessity to have an excess of cadmium is in agreement with what we have learned from the existing preparation methods of CdSe NPLs ${ }^{9,12,25}$ and can be rationalized with the two $\mathrm{Cd}$-rich facets present in the $\mathrm{ZB}$ NPLs. ${ }^{28}$

In addition to cadmium octanoate, we have investigated longer cadmium carboxylates such as cadmium decanoate $\left(\mathrm{CdC}_{10}\right)$ and cadmium myristate $\left(\mathrm{CdC}_{14}\right)$. The reactivity of $\mathrm{CdC}_{10}$ is close to that of $\mathrm{CdC}_{8}$, and a simple replacement of $\mathrm{CdC}_{8}$ with $\mathrm{CdC}_{10}$ in the synthesis route does not seem to affect the chemical yield and the quality of the NPLs. When $\mathrm{CdC}_{14}$ is used, however, NPLs are formed but with a yield that is much lower. After size selective precipitation, three-ML NPLs are identified with other structures in the TEM image, but they appear as very irregular wormlike structures (Figure 4b).

To better understand the formation mechanism, we followed the formation of CdSe NPLs during the reaction by repeating our synthesis of NPLs with cadmium octanoate using TOPSe $(2.24 \mathrm{M})$ and acquiring absorption and TEM images of aliquot samples at different times during the reaction (Figure 5).

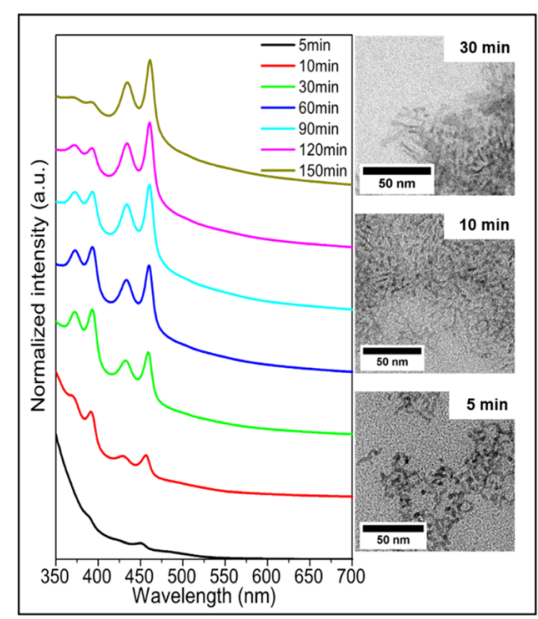

Figure 5. Absorption spectra and TEM images of CdSe NPLs at different reaction times: $1 \mathrm{mmol}$ of $\mathrm{CdC}_{8}$ in $10 \mathrm{~mL}$ of ODE and 100 $\mu \mathrm{L}$ of TOPSe $(2.24 \mathrm{M})$ in $0.5 \mathrm{~mL}$ of ODE injected at $175^{\circ} \mathrm{C}$ under Ar.

Five minutes after the injection of the Se precursor, the absorption spectrum of the aliquot shows two small peaks at 380 and $450 \mathrm{~nm}$ that are characteristics of two-ML and threeML thick NPLs, respectively, with some lateral confinement. The TEM images do not clearly display the NPLs but rather show the aggregates that look much more like a mesh of connected wormlike structures. It is very striking that at this point, any excitonic structures appear at all in the absorption spectra. These strongly suggest that at least part of this wormlike structure has a precisely controlled thickness of two 

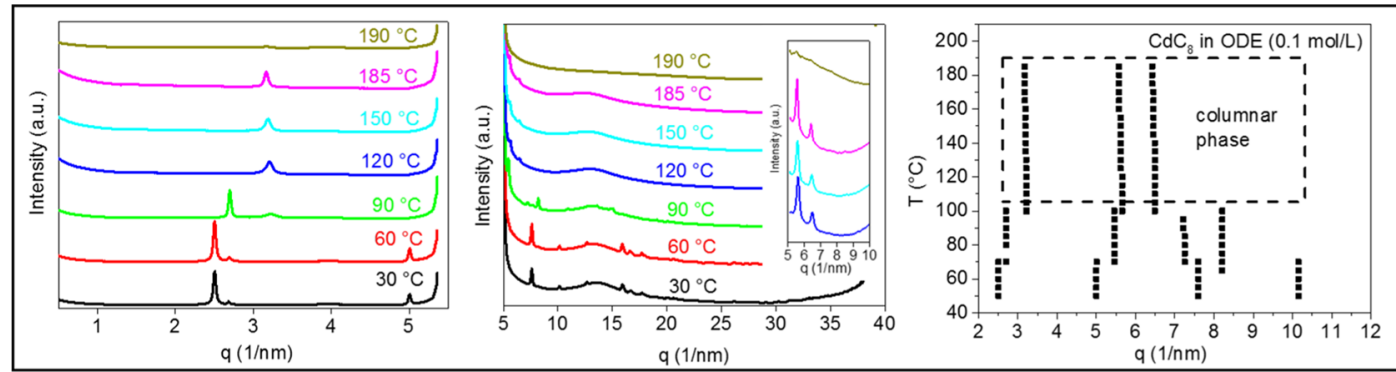

Figure 6. SAXS/WAXS patterns acquired during the heating of the precursor solution $\left(\mathrm{CdC}_{8}\right.$ in $\left.\mathrm{ODE}, \mathrm{C}_{\mathrm{CdC}_{8}}=0.1 \mathrm{~mol} / \mathrm{L}\right)$ from 30 to $190{ }^{\circ} \mathrm{C}$ and its corresponding plot of temperature vs $q\left(\mathrm{~nm}^{-1}\right)$.

and three CdSe MLs. The connectivity of the TEM images suggests that small nanocrystals aggregate possibly through the oriented attachment, but it is also possible that geometrical constraints imposed by the bulky $\mathrm{C} 8$ chain of the carboxylate prevent the structure to extend laterally as easily as when tiny cadmium acetate is present in solution.

After $10 \mathrm{~min}$, the excitonic peaks that we identified at $5 \mathrm{~min}$ are clearly present, slightly red-shifted, and more pronounced. The red shift is consistent with the lateral extension of the $2 \mathrm{D}$ NPLs so that the lateral confinement of the exciton decreases. Two additional peaks corresponding to the light hole-electron transitions for two- and three-ML NPLs are now clearly visible. The TEM examination shows rodlike structures with lateral dimensions of few nanometers. At this stage, it is still very hard to assert with TEM that NPLs are formed. The absorption spectra, however, unambiguously show that the sample contains 2D structures with precisely defined thicknesses of two and three CdSe MLs. We thus conclude that most of these rodlike structures have very well-defined thicknesses, slightly similar to wires. As the reaction proceeds, we observe a shape refinement process during the lateral growth as the initial small undefined NPLs slowly grew into well-defined rectangular-shaped NPLs (e.g., TEM image at $30 \mathrm{~min}$ ). At this stage, the absorption spectra of the aliquot show the characteristics of two-ML and three-ML thick NPLs with positions at 390 and $460 \mathrm{~nm}$, showing that the lateral dimensions of the NPLs are larger than $7 \mathrm{~nm}$, the Bohr diameter of the $2 \mathrm{D}$ exciton. ${ }^{29}$ As the reaction proceeds at $175{ }^{\circ} \mathrm{C}$, we observe on the absorption spectra that the two-ML thick population disappears, whereas the three-ML population continues to grow steadily. This means that the growth of NPLs occurs not only laterally to gain a well-defined shape but also that under these conditions, two-ML thick NPLs are not stable and dissolve. After $2.5 \mathrm{~h}$, almost all the two-ML NPLs were converted to three MLs. This is in agreement with the growth model that predicts that a rapid growth of two-ML NPLs would eventually lead to slow formation of thicker NPLs at high temperature and long reaction times. ${ }^{24}$

The TEM pictures observed in Figure 5 combined with the absorption spectra taken during the NPL formation show that ill-defined wormlike structures possess absorption features that correspond precisely to the absorption of colloidal structures with $1 \mathrm{D}$ confinement. How do those wormlike structures form and how they can transform into well-defined NPLs remain an open question. We could not find any evidence of single dot formation, and these $2 \mathrm{D}$ embryos are very different from the ones observed recently in Chen et al. ${ }^{18}$ As we mentioned earlier, in our case, the mixture composed of cadmium octanoate and TOPSe is perfectly soluble in ODE under the reaction conditions used, and no aggregation can be observed.
To check if soft templating of $\mathrm{CdC}_{8}$ in the ODE hydrocarbon chain could play a role in the formation of NPLs at least in the early stages, we performed SAXS and WAXS analyses of $\mathrm{CdC}_{8}$ in ODE at different temperatures. By heating the precursor solution (e.g., $C_{\mathrm{CdC}_{8}}=0.1 \mathrm{~mol} / \mathrm{L}$ shown in Figure 6) and recording SAXS/WAXS patterns at different temperatures, we measured peak locations corresponding to each temperature ramp and plotted diagrams of temperature versus wave vector $q$ $\left(\mathrm{nm}^{-1}\right)$. On the basis of these diagrams, we were able to determine the appearance of liquid-crystal phases of $\mathrm{CdC}_{8}$ and their transition temperatures. We observe that $\mathrm{CdC}_{8}$ forms columnar structures in ODE that can exist up to $\approx 185^{\circ} \mathrm{C}$ for a concentration of $C_{\mathrm{CdC}_{8}}=0.1 \mathrm{~mol} / \mathrm{L}$, but there is no signal corresponding to any mesophase acquired above $190{ }^{\circ} \mathrm{C}$. In spite of this absence of any ordered structures above $190{ }^{\circ} \mathrm{C}$, we could synthesize CdSe NPLs with an injection of TOPSe at $200{ }^{\circ} \mathrm{C}$ and even $225^{\circ} \mathrm{C}$ (Figure 2). This excludes the necessity of a lamellar structure for synthesizing CdSe NPLs in the absence of acetate salts. Complementary SAXS/WAXS experiment (Figure S4) shows that lowering $C_{\mathrm{CdC}_{8}}$ to $0.005 \mathrm{~mol} / \mathrm{L}$ causes the formation of a lamellar structure that exists up to 180 ${ }^{\circ} \mathrm{C}$, as indicated by the peaks in the scattering patterns at wave vectors $q^{*}, 2 q^{*}$, and $3 q^{*}\left(2.64,5.35\right.$, and $\left.8.02 \mathrm{~nm}^{-1}\right)$. This means that the phase behavior of the cadmium carboxylate in ODE depends on its concentration in the organic solvent. In the case of $\mathrm{CdC}_{10}$ and $\mathrm{CdC}_{14}$ at a concentration of $0.1 \mathrm{~mol} / \mathrm{L}$ in ODE, both precursor solutions do not show mesophase transitions before melting into the liquid phase (Figure S5) at temperatures higher than $100{ }^{\circ} \mathrm{C}$. Similarly, NPLs could be obtained with $\mathrm{CdC}_{10}$ in ODE and TOPSe injected at temperatures higher than $100{ }^{\circ} \mathrm{C}$. This analysis confirms that CdSe NPLs can be synthesized in a completely soluble mixture without acetate.

What could then be the driving mechanism for the formation of 2D structures? We have shown that soft templating could be ruled out in our case. We also do not observe in TEM small seeds together with NPL as previously shown in the case of oriented attachment. Finally, the recent formation mechanism driven by insolubility is not consistent with the fact that we do not observe an insoluble material in our solution during the onset of NPL formation. We suggest that ligand packing density and steric hindrance due to packing could play a significant role for the formation of ZB CdSe NPLs. Indeed, we observe that in the absence of short-chain cadmium ligands, the NPLs form as elongated, thin, wirelike structures without uniform lateral extension. We propose that as it has been discussed for $\mathrm{CdSe}$ magic-sized clusters, ${ }^{30}$ the packing density of long alkyl chain carboxylate on the cadmium-rich [100] 
facets (the larger surfaces of the NPLs) cannot match the density of cadmium atoms on these surfaces. Because NPLs have a large amount of cations $(n+1$ cadmium planes for $n$ selenium planes), they require negatively charged ligands to counterbalance their positive charge. This balance equilibrium can be obtained if two carboxylates can bind every cadmium atom in excess. We hypothesize that this can be done only if short-chain ligands such as cadmium acetate are present during the synthesis. When cadmium acetate is added in the synthesis with $\mathrm{CdC}_{8}$, indeed large $2 \mathrm{D}$ objects are obtained with unrestricted lateral extension (Figure S6). We also found that by simply adjusting the chain length of short carboxylate ligands, it is possible to modify the steric hindrance and ligand packing under the same experimental conditions, thus tuning the lateral extension of the ZB CdSe NPLs (Figure S7). Further studies are in progress to test this hypothesis.

\section{CONCLUSIONS}

In conclusion, we show that it is possible to synthesize CdSe NPLs from pure long cadmium carboxylate without the addition of any short-chain ligands. The effects of several experimental parameters are studied. We hypothesize that the steric hindrance caused by ligand packing should affect the growth of NPLs. Such a hypothesis could lead to a better understanding of the formation mechanism of $2 \mathrm{D}$ nanostructures. Our study highlights the diversity of formation mechanisms that can lead to the NPL depending on the reaction conditions and the type of the precursor used.

\section{EXPERIMENTAL SECTION}

Chemicals. Cadmium acetate dihydrate $\left[\mathrm{Cd}(\mathrm{OAc})_{2} \cdot 2\right.$ $\left(\mathrm{H}_{2} \mathrm{O}\right)$, Sigma-Aldrich, 98\%], oleic acid (OA, Sigma-Aldrich, 90\%), ODE (Sigma-Aldrich, 90\%), hexadecane (Sigma-Aldrich, 98\%), trioctylphosphine (TOP, Cytec, 90\%), selenium powder (Sigma-Aldrich, 99.5\%), sodium octanoate (Sigma-Aldrich, 99\%), sodium myristate (Sigma-Aldrich, 99\%), cadmium nitrate tetrahydrate (Sigma-Aldrich, 98\%), cadmium oxide (SigmaAldrich, 99.99\%), decanoic acid (Sigma-Aldrich, 98\%), acetic acid (Sigma-Aldrich, 99.85\%), propionic acid (Sigma-Aldrich, 99.5\%), butyric acid (Sigma-Aldrich, 99\%), n-hexane (VWR, 95\%), and ethanol (Carlo Erba, 99.8\%) are purchased for the synthesis of NPLs and used without any further purification.

Synthesis of Cadmium Octanoate $\mathbf{C d C}_{8}$. Cadmium nitrate $(1.23 \mathrm{~g})$ is dissolved in $10 \mathrm{~mL}$ of distilled water, and $2.08 \mathrm{~g}$ of sodium octanoate is dissolved in $100 \mathrm{~mL}$ of distilled water by stirring and sonication. Once two transparent solutions are obtained, they are mixed and stirred for $30 \mathrm{~min}$ at room temperature. The resulting white precipitate of $\mathrm{CdC}_{8}$ is filtered, rinsed three times using distilled water, and then dried under vacuum overnight.

Synthesis of Cadmium Myristate $\mathbf{C d C}_{14}$. Cadmium nitrate $(1.23 \mathrm{~g})$ is dissolved in $10 \mathrm{~mL}$ of methanol, and 3.13 $\mathrm{g}$ of sodium myristate is dissolved in $100 \mathrm{~mL}$ of methanol by stirring and sonication. Once the two transparent solutions are obtained, they are mixed and stirred for $30 \mathrm{~min}$ at room temperature. The resulting white precipitate of $\mathrm{CdC}_{14}$ is filtered, rinsed three times using methanol, and then dried under vacuum overnight.

Synthesis of Cadmium Decanoate $\mathrm{CdC}_{10}$. In a threeneck flask, $1 \mathrm{~g}$ of cadmium oxide, $4.03 \mathrm{~g}$ of decanoic acid, and $20 \mathrm{~mL}$ of ODE are degassed under vacuum for $20 \mathrm{~min}$ at room temperature. The mixture is then heated at $240{ }^{\circ} \mathrm{C}$ under argon flow for $30 \mathrm{~min}$. When a colorless solution is obtained, the solution is cooled down to around $60{ }^{\circ} \mathrm{C}$ and $50 \mathrm{~mL}$ of hexane is added to the flask. The resulting white precipitate of $\mathrm{CdC}_{10}$ is filtered, rinsed three times using ethanol, and then dried under vacuum overnight.

Synthesis of Se-ODE Solution (0.1 M). In a three-neck flask, $140 \mathrm{~mL}$ of octadecene is degassed for $30 \mathrm{~min}$ before a solution of $1.18 \mathrm{~g}$ of selenium dispersed in $10 \mathrm{~mL}$ of octadecene is injected with the temperature increased from 180 to $205^{\circ} \mathrm{C}$ under argon. The solution is then heated for $30 \mathrm{~min}$ at $205^{\circ} \mathrm{C}$.

Synthesis of Stoichiometric TOPSe (2.24 M). In a glovebox, $2.65 \mathrm{~g}$ of selenium powder is dissolved by agitation in a flask with $15 \mathrm{~mL}$ of TOP for $72 \mathrm{~h}$. The transparent greenish solution obtained is stored inside the glovebox, and a desired amount of TOPSe is collected for injection just before the reactions.

Hot-Injection Synthesis of CdSe NPLs Emitting at 460 $\mathrm{nm}$ with TOPSe (2.24 M). In a three-neck flask, $0.40 \mathrm{~g}$ of cadmium octanoate and $10 \mathrm{~mL}$ of ODE are degassed under vacuum for $20 \mathrm{~min}$. The flask is heated up to $175{ }^{\circ} \mathrm{C}$ under argon flow, and then a homogenous mixture of $100 \mu \mathrm{L}$ stoichiometric TOPSe and $0.5 \mathrm{~mL} \mathrm{ODE}$ is injected. The synthesis reaction lasts for $2 \mathrm{~h}$, and $2 \mathrm{~mL}$ of $\mathrm{OA}$ is injected. The as-synthesized CdSe NPLs are precipitated, washed three times with ethanol, and then suspended in hexane.

One-Pot Synthesis of CdSe NPLs with Metallic Selenium Powder. In a three-neck flask, $0.40 \mathrm{~g}$ of cadmium octanoate and $10 \mathrm{~mL}$ of ODE are degassed under vacuum for $20 \mathrm{~min}$. Under argon flow, a homogeneous mixture of $100 \mu \mathrm{L}$ stoichiometric TOPSe and $0.5 \mathrm{~mL}$ ODE is injected before the flask is heated up to $175^{\circ} \mathrm{C}$. The synthesis reaction lasts for $2 \mathrm{~h}$ and $2 \mathrm{~mL}$ of OA is injected. The as-synthesized CdSe NPLs are precipitated, washed three times with ethanol, and then suspended in hexane.

Material Characterizations. Optical absorption and PL spectroscopy are performed using a UV visible spectrometer (Shimadzu, UV3600) and a PL spectrometer (Jobin-Yvon HORIBA, Fluoromax-3). TEM imaging is done using JEOL 2010.

Quantum Yield Measurement. The quantum yield (QY) of as-synthesized three-ML CdSe NPLs was measured using a comparative method with an organic fluorophore (rhodamine 6G). Absorption and emission spectra were acquired at different concentrations, and QY was calculated following the equation:

$$
\mathrm{QY}=\mathrm{QY}_{\mathrm{ref}}\left(\frac{n^{2}}{n_{\mathrm{ref}}^{2}}\right)\left(\frac{I}{A}\right)\left(\frac{A_{\text {ref }}}{I_{\text {ref }}}\right)
$$

where $n$ is the refractive index of the solvent, $I$ is the integrated fluorescence intensity, and $A$ is the absorbance at the excitation wavelength.

Small-/Wide-Angle X-ray Scattering. SAXS/WAXS experiments were performed at the ID2 beamline at the ESRF (European Synchrotron Radiation Facility, Grenoble, France) using an in-capillary heating-up method.

\section{ASSOCIATED CONTENT}

\section{Supporting Information}

The Supporting Information is available free of charge on the ACS Publications website at DOI: 10.1021/acsomega.8b01006.

XRD patterns of CdSe NPLs; TEM images of CdSe nanowires synthesized using TOPSe ( $1 \mathrm{M})$; SAXS/ 
WAXS patterns acquired during the heating of the precursor solution $\left(\mathrm{CdC}_{8}, \mathrm{CdC}_{10}\right.$, and $\left.\mathrm{CdC}_{14}\right)$ with their corresponding plots of temperature versus $q\left(\mathrm{~nm}^{-1}\right)$; absorption spectrum and TEM images of CdSe NPLs synthesized from $\mathrm{CdC}_{8}$ with $\mathrm{Cd}(\mathrm{Ac})_{2}$; and influence of short carboxylate ligands on the lateral extension of the CdSe NPLs (PDF)

\section{AUTHOR INFORMATION}

\section{Corresponding Authors}

*E-mail: benjamin.abecassis@ens-lyon.fr (B.A.).

*E-mail: benoit.dubertret@espci.fr (B.D.).

\section{ORCID}

Benjamin Abécassis: 0000-0002-1629-9671

\section{Notes}

The authors declare no competing financial interest.

\section{ACKNOWLEDGMENTS}

This work was supported by the European Commission via the Marie-Sklodowska Curie action Phonsi (H2020-MSCA-ITN642656). We thank the ESRF for the provision of beam time and S. Prévost for the assistance provided during these experiments.

\section{REFERENCES}

(1) Yang, J.; Son, J. S.; Yu, J. H.; Joo, J.; Hyeon, T. Advances in the Colloidal Synthesis of Two-Dimensional Semiconductor Nanoribbons. Chem. Mater. 2013, 25, 1190-1198.

(2) Nasilowski, M.; Mahler, B.; Lhuillier, E.; Ithurria, S.; Dubertret, B. Two-Dimensional Colloidal Nanocrystals. Chem. Rev. 2016, 116, 10934-10982.

(3) Ithurria, S.; Dubertret, B. Quasi 2D Colloidal CdSe Platelets with Thicknesses Controlled at the Atomic Level. J. Am. Chem. Soc. 2008, 130, 16504-16505.

(4) Ithurria, S.; Tessier, M. D.; Mahler, B.; Lobo, R. P. S. M.; Dubertret, B.; Efros, A. L. Colloidal Nanoplatelets with TwoDimensional Electronic Structure. Nat. Mater. 2011, 10, 936-941.

(5) Fox, M.; Bertsch, G. F. Optical Properties of Solids. Am. J. Phys. 2002, 70, 1269-1270.

(6) Rodina, A. V.; Efros, A. L. Effect of Dielectric Confinement on Optical Properties of Colloidal Nanostructures. J. Exp. Theor. Phys. 2016, 122, 554-566.

(7) Benchamekh, R.; Gippius, N. A.; Even, J.; Nestoklon, M. O.; Jancu, J.-M.; Ithurria, S.; Dubertret, B.; Efros, A. L.; Voisin, P. TightBinding Calculations of Image-Charge Effects in Colloidal Nanoscale Platelets of CdSe. Phys. Rev. B: Condens. Matter Mater. Phys. 2014, 89, 035307.

(8) Tessier, M. D.; Javaux, C.; Maksimovic, I.; Loriette, V.; Dubertret, B. Spectroscopy of Single CdSe Nanoplatelets. ACS Nano 2012, 6, 6751-6758.

(9) Liu, Y.-H.; Wang, F.; Wang, Y.; Gibbons, P. C.; Buhro, W. E. Lamellar Assembly of Cadmium Selenide Nanoclusters into Quantum Belts. J. Am. Chem. Soc. 2011, 133, 17005-17013.

(10) Son, J. S.; Yu, J. H.; Kwon, S. G.; Lee, J.; Joo, J.; Hyeon, T. Colloidal Synthesis of Ultrathin Two-Dimensional Semiconductor Nanocrystals. Adv. Mater. 2011, 23, 3214-3219.

(11) Joo, J.; Son, J. S.; Kwon, S. G.; Yu, J. H.; Hyeon, T. LowTemperature Solution-Phase Synthesis of Quantum Well Structured CdSe Nanoribbons. J. Am. Chem. Soc. 2006, 128, 5632-5633.

(12) Son, J. S.; Wen, X.-D.; Joo, J.; Chae, J.; Baek, S.-i.; Park, K.; Kim, J. H.; An, K.; Yu, J. H.; Kwon, S. G.; et al. Large-Scale Soft Colloidal Template Synthesis of $1.4 \mathrm{Nm}$ Thick Cdse Nanosheets. Angew. Chem., Int. Ed. 2009, 48, 6861-6864.
(13) Maiti, P. S.; Sadan, M. B. Tuning the Surface Properties of Alloyed CdS X Se 1-x 2D Nanosheets. RSC Adv. 2015, 5, 100834100837.

(14) Bouet, C.; Mahler, B.; Nadal, B.; Abecassis, B.; Tessier, M. D.; Ithurria, S.; Xu, X.; Dubertret, B. Two-Dimensional Growth of CdSe Nanocrystals, from Nanoplatelets to Nanosheets. Chem. Mater. 2013, $25,639-645$.

(15) Li, Z.; Qin, H.; Guzun, D.; Benamara, M.; Salamo, G.; Peng, X. Uniform Thickness and Colloidal-Stable CdS Quantum Disks with Tunable Thickness: Synthesis and Properties. Nano Res. 2012, 5, 337351.

(16) Ithurria, S.; Bousquet, G.; Dubertret, B. Continuous Transition from 3D to 1D Confinement Observed during the Formation of CdSe Nanoplatelets. J. Am. Chem. Soc. 2011, 133, 3070-3077.

(17) Schliehe, C.; Juarez, B. H.; Pelletier, M.; Jander, S.; Greshnykh, D.; Nagel, M.; Meyer, A.; Foerster, S.; Kornowski, A.; Klinke, C.; et al. Ultrathin $\mathrm{PbS}$ Sheets by Two-Dimensional Oriented Attachment. Science 2010, 329, 550-553.

(18) Chen, Y.; Chen, D.; Li, Z.; Peng, X. Symmetry-Breaking for Formation of Rectangular CdSe Two-Dimensional Nanocrystals in Zinc-Blende Structure. J. Am. Chem. Soc. 2017, 139, 10009-10019.

(19) Lyashchova, A.; Dmytruk, A.; Dmitruk, I.; Klimusheva, G.; Mirnaya, T.; Asaula, V. Optical Absorption, Induced Bleaching, and Photoluminescence of CdSe Nanoplatelets Grown in Cadmium Octanoate Matrix. Nanoscale Res. Lett. 2014, 9, 88.

(20) Adeosun, S. O.; Ellis, H. A. Binary Phase Diagrams of Some Bivalent Metal Carboxylate Systems. Thermochim. Acta 1979, 28, 313321.

(21) Nelson, P. N.; Taylor, R. A. Theories and Experimental Investigations of the Structural and Thermotropic Mesomorphic Phase Behaviors of Metal Carboxylates. Appl. Petrochem. Res. 2014, 4, 253285.

(22) Konkoly-Thege, I.; Ruff, I. A Quantitative Differential Thermal Analysis Study of Phase Transitions in Some Zinc and Cadmium Carboxylates. Thermochim. Acta 1978, 24, 89-96.

(23) Abécassis, B.; Bouet, C.; Garnero, C.; Constantin, D.; Lequeux, N.; Ithurria, S.; Dubertret, B.; Pauw, B. R.; Pontoni, D. Real-Time in Situ Probing of High-Temperature Quantum Dots Solution Synthesis. Nano Lett. 2015, 15, 2620-2626.

(24) Riedinger, A.; Ott, F. D.; Mule, A.; Mazzotti, S.; Knüsel, P. N.; Kress, S. J. P.; Prins, F.; Erwin, S. C.; Norris, D. J. An Intrinsic Growth Instability in Isotropic Materials Leads to Quasi-Two-Dimensional Nanoplatelets. Nat. Mater. 2017, 16, 743-748.

(25) Li, Z.; Peng, X. Size/Shape-Controlled Synthesis of Colloidal CdSe Quantum Disks: Ligand and Temperature Effects. J. Am. Chem. Soc. 2011, 133, 6578-6586.

(26) Pedetti, S.; Nadal, B.; Lhuillier, E.; Mahler, B.; Bouet, C.; Abécassis, B.; Xu, X.; Dubertret, B. Optimized Synthesis of CdTe Nanoplatelets and Photoresponse of CdTe Nanoplatelets Films. Chem. Mater. 2013, 25, 2455-2462.

(27) Bullen, C.; Van Embden, J.; Jasieniak, J.; Cosgriff, J. E.; Mulder, R. J.; Rizzardo, E.; Gu, M.; Raston, C. L. High Activity Phosphine-Free Selenium Precursor Solution for Semiconductor Nanocrystal Growth. Chem. Mater. 2010, 22, 4135-4143.

(28) Chen, D.; Gao, Y.; Chen, Y.; Ren, Y.; Peng, X. Structure Identification of Two-Dimensional Colloidal Semiconductor Nanocrystals with Atomic Flat Basal Planes. Nano Lett. 2015, 15, 44774482.

(29) Efros, A. L.; Rosen, M.; Kuno, M.; Nirmal, M.; Norris, D. J.; Bawendi, M. Band-Edge Exciton in Quantum Dots of Semiconductors with a Degenerate Valence Band: Dark and Bright Exciton States. Phys. Rev. B: Condens. Matter Mater. Phys. 1996, 54, 4843-4856.

(30) Beecher, A. N.; Yang, X.; Palmer, J. H.; LaGrassa, A. L.; Juhas, P.; Billinge, S. J. L.; Owen, J. S. Atomic Structures and Gram Scale Synthesis of Three Tetrahedral Quantum Dots. J. Am. Chem. Soc. 2014, 136, 10645-10653. 